\title{
In vivo Fluorescence Imaging of Extracellular ATP in the Mouse Cerebral Cortex with a Hybrid-type Optical Sensor
}

Nami Kitajima ${ }^{1}$, Kenji Takikawa' ${ }^{1}$, Hiroshi Sekiya ${ }^{2}$, Daisuke Asanuma ${ }^{1}$, Hirokazu Sakamoto ${ }^{1}$, Shigeyuki Namiki ${ }^{1}$, Masamitsu lino ${ }^{3}$ and Kenzo Hirose ${ }^{1,4, *}$

1Department of Pharmacology, Graduate School of Medicine, The University of Tokyo, Tokyo, Japan; 2Department of Physiology, Graduate School of Medicine, The University of Tokyo, Tokyo, Japan; ${ }^{3}$ Department of Cellular and Molecular Pharmacology, Nihon University School of Medicine, Tokyo, Japan; ${ }^{4}$ International Research Center for Neurointelligence, The University of Tokyo, Tokyo, Japan

*For correspondence: kenzoh@m.u-tokyo.ac.jp

[Abstract] Adenosine 5'-triphosphate (ATP) works as an extracellular signaling molecule for cells in the brain, such as neurons and glia. Cellular communication via release of ATP is involved in a range of processes required for normal brain functions, and aberrant communication is associated with brain disorders. To investigate the mechanisms underlying these cellular processes, various techniques have been developed for the measurement of extracellular ATP. To monitor the dynamics of extracellular ATP signaling with high spatiotemporal resolution, we recently developed a hybrid-type ATP optical sensor (ATPOS) that enables in vivo fluorescence imaging of extracellular ATP dynamics in the brain. ATPOS is synthesized by labeling an ATP-binding protein, Bacillus FoF 1 -ATP synthase $\varepsilon$ subunit, with a smallmolecular fluorescent dye Сy3. Injection of ATPOS into the cerebral cortex of living mice enables visualization of the wave-like propagation of extracellular ATP release in response to electrical stimulation. The protocol described here should be useful for visualizing ATP signaling in diverse processes involved in intercellular communication in the brain.

Keywords: ATP, Extracellular signaling molecule, In vivo imaging, Fluorescent sensor, Brain

[Background] Adenosine 5'-triphosphate (ATP), which is well known as the intracellular energy currency, works as an extracellular signaling molecule in various organs and tissues of the body (Zimmermann, 2016). In the brain, ATP is released from neurons and glia (Pankratov et al., 2006; Butt, 2011), and is involved in neurotransmission (Burnstock, 2007), neuron-glia interaction (Fields and Burnstock, 2006; Khakh and North, 2012), and regulation of blood flow (MacVicar and Newman, 2015). Release of ATP also occurs during pathological neuronal activity known as cortical spreading depression (CSD) (Schock et al., 2007; Heinrich et al., 2012), which is associated with migraine and cerebral ischemia (Lauritzen et al., 2011). Thus, extracellular ATP signaling is considered to play key roles not only in normal brain functions but also in brain disorders.

To better understand the roles of ATP signaling, it is important to visualize the spatiotemporal changes of extracellular ATP. Various techniques have been developed for monitoring extracellular ATP; for example, bioluminescence assays combined with microdialysis and electrophysiological methods using enzyme-coated electrodes can quantitate extracellular ATP in the brain of living animals (Gourine et al., 
2005; Melani et al., 2005). However, these techniques provide little spatial information. In contrast to these non-imaging techniques, fluorescence imaging using optical sensors is a promising tool for monitoring the dynamics of a target molecule with high spatiotemporal resolution (Giepmans et al., 2006). To date, a variety of ATP sensors based on fluorescent proteins have been reported (Berg et al., 2009; Imamura et al., 2009; Tantama et al., 2013; Yaginuma et al., 2015; Arai et al., 2018; Lobas et al., 2019). However, their application in in vivo imaging of extracellular ATP remains difficult (Conley et al., 2017; Lobas et al., 2019) because they are not sensitive enough to detect ATP released into the extracellular space (Yegutkin, 2008). Moreover, these ATP sensors exhibit pH-dependent fluorescence changes due to the $\mathrm{pH}$ sensitivity of the fluorescent proteins used in the sensors (Tsien, 1998; Chudakov et al., 2010). This $\mathrm{pH}$ dependence is unfavorable for application in the brain, where the tissue $\mathrm{pH}$ fluctuates during neuronal activity (Chesler and Kaila, 1992; Magnotta et al., 2012).

We recently developed a hybrid-type ATP optical sensor, ATPOS, which consists of an ATP-binding

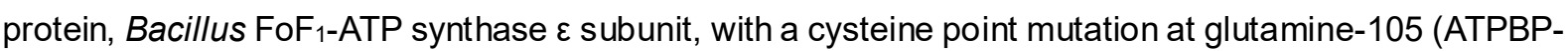
Q105C) and a small-molecular fluorescent dye Cy3 bearing a cysteine-reactive maleimide group (Kitajima et al., 2020). ATPOS shows a large fluorescence response to ATP ( 2 fold) with high affinity $(K \mathrm{~d}=\sim 150 \mathrm{nM}), \mathrm{pH}$ independence, and high selectivity, and is therefore suitable for fluorescence imaging of extracellular ATP dynamics (Kitajima et al., 2020). For application of ATPOS to extracellular ATP imaging in the brain, we used BoNT/C-Hc, a nontoxic subunit of Clostridium botulinum type $\mathrm{C}$ neurotoxin (Tsukamoto et al., 2005), through which ATPOS can be anchored to the surface of neuronal cell membranes. In addition, we adopted Alexa Fluor 488 (Alexa488) as a reference fluorophore, so that ATPOS can be used for dual-color ratiometric imaging. Accordingly, ATPOS is applied as a form of molecular complex with BoNT/C-Hc and Alexa488-labeled streptavidin (Figure 1). After delivery into the cerebral cortex of living mice, the ATPOS complex can aid visualization of wave-like extracellular ATP dynamics during CSD evoked by electrical stimulation (Kitajima et al., 2020). Here, we describe the protocols for the production of ATPOS and its application to fluorescence imaging of extracellular ATP in the mouse brain. These protocols will be useful for studying the roles of extracellular ATP signaling in various physiological and pathological processes in the brain. 


\section{bĭo-protocol

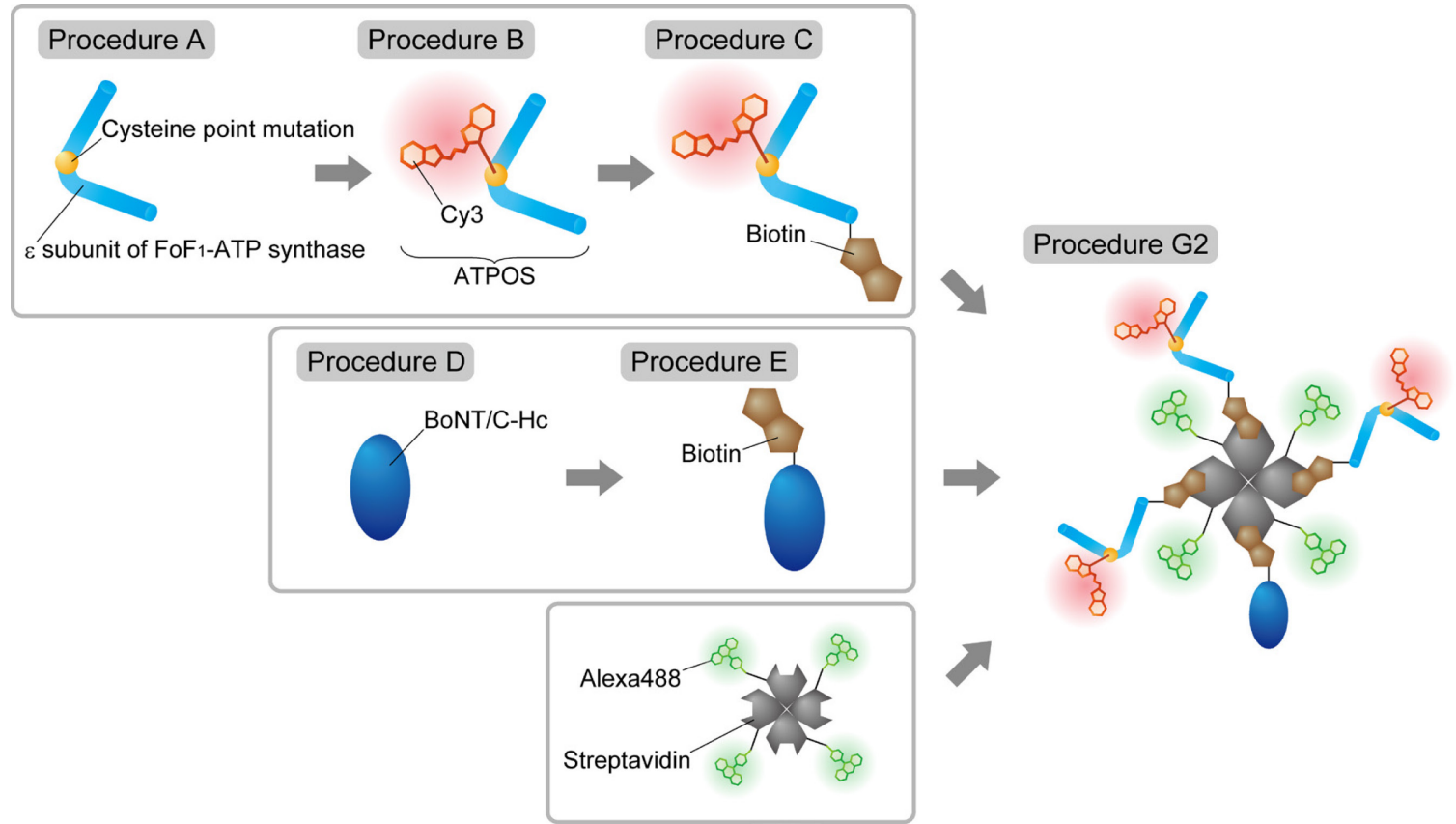

Figure 1. Schematic illustration of the experimental workflow for the production of the ratiometric ATPOS complex. Procedure A: The $\varepsilon$ subunit of FoF1-ATP synthase with a cysteine point mutation at glutamine-105 is expressed in bacterial cells. Procedure B: ATPOS is produced by labeling the $\varepsilon$ subunit with Cy3 bearing a cysteine-reactive maleimide group. Procedure C: Biotin is covalently attached to ATPOS. Procedure D: BoNT/C-Hc is expressed in bacterial cells. Procedure E: Biotin is covalently attached to BoNT/C-Hc. Procedure G2: Biotinylated ATPOS, biotinylated BoNT/C-Hc, and Alexa488-labeled streptavidin are mixed at a molar ratio of 3:1:1 to assemble the ratiometric ATPOS complex via biotin-streptavidin linkage.

\section{Materials and Reagents}

1. HiTrap TALON crude 1-ml column (BD Biosciences, catalog number: 29048565)

2. TALON metal affinity resin (Clontech, catalog number: 635503)

3. PD-10 desalting column (GE Healthcare, catalog number: 17085101)

4. Empty disposable PD-10 column (GE Healthcare, catalog number: 17043501)

5. Amicon Ultra-0.5 centrifugal filter unit (10 kDa cutoff) (Merck, catalog number: UFC5010)

6. Amicon Ultra-0.5 centrifugal filter unit (30 kDa cutoff) (Merck, catalog number: UFC5030)

7. Dental acrylic (GC, Fuji LUTE BC)

8. Glass capillary (Harvard Apparatus, catalog number: 30-0053)

9. 10-ml syringe (Terumo, catalog number: SS-10LZ)

10. Plastic tube (Imamura, catalog number: 01605)

11. Monopolar tungsten microelectrode (FHC, catalog number: UEWMGCSEKNNM)

12. Escherichia coli BL21 (DE3)

13. Wild-type mice (C57BL/6NCrSlc, male, postnatal 1-2 months old)

14. Plasmid encoding ATPBP-Q105C (Kitajima et al., 2020) 
15. Plasmid encoding BoNT/C-Hc (Takikawa et al., 2014)

16. Cy3 maleimide (GE Healthcare, catalog number: PA13131)

17. Alexa488-labeled streptavidin (Thermo Scientific, catalog number: S11223)

18. NHS-PEG 4 -biotin (Thermo Scientific, catalog number: A39259)

19. Ampicillin (Nacalai Tesque, catalog number: 02739-32)

20. Kanamycin (Nacalai Tesque, catalog number: 19839-44)

21. Isopropyl- $\beta$-D-thiogalactopyranoside (IPTG) (Nacalai Tesque, catalog number: 19742-81)

22. Lysozyme (Nacalai Tesque, catalog number: 19499-91)

23. Triton X100 (Nacalai Tesque, catalog number: 12967-45)

24. DNase I (Takara, catalog number: 2270A)

25. Imidazole (Nacalai Tesque, catalog number: 19004-35)

26. Medetomidine (Nippon Zenyaku, Domitor)

27. Midazolam (Sandoz, Midazolam Injection $10 \mathrm{mg}$ [SANDOZ])

28. Butorphanol (Meiji Seika Pharma, Vetorphale)

29. Mineral oil (Sigma, catalog number: M8410)

30. Dimethyl sulfoxide (DMSO) (Nacalai Tesque, catalog number: 13408-64)

31. Luria Bertani (LB) agar plates (see Recipes)

32. $2 \times$ YT media (see Recipes)

33. LB media (see Recipes)

34. Phosphate-buffered saline (PBS) (see Recipes)

35. 10× PBS (see Recipes)

36. Artificial cerebrospinal fluid (aCSF) (see Recipes)

37. HEPES-buffered saline (HBS) (see Recipes)

38. Anesthetic solution (see Recipes)

\section{Equipment}

1. 1-L flask

2. French press (Aminco, model: FA-080B)

3. Fast protein liquid chromatography system (GE Healthcare, model: AKTA purifier 10)

4. Spectrophotometer (Eppendorf, model: Eppendorf BioPhotometer Plus)

5. Heating pad (Bio Research Center, model: BWT-100A)

6. Metal frame and frame holder (custom-made, Figures 2A and 2B)

7. Dental drill (Nakanishi, model: ULTIMATE XL)

8. Pipette puller (Sutter Instrument, model: P-97)

9. Beveller (PRIME TECH, model: EZP-75)

10. Wide-field microscope (Leica, model: M165FC)

11. Objective lens (Leica, Plan APO 1.0×, catalog number: 10450028)

12. Filter set (Leica, Filter set ET GFP3/CY3, catalog number: 10450612) 
13. High-speed scanning polychromatic light source (Hamamatsu Photonics, model: C7773)

14. EM-CCD camera (TELEDYNE Photometrics, model: Evolve512)

15. Stereotaxic manipulator (Luigs and Neumann, model: SM-5)

16. Stimulus isolator (AMPI, model: ISO-Flex)

\section{Software}

1. ImageJ/Fiji (NIH, version: $1.50 \mathrm{e}$, https://imagej. net/Fiji)

2. MetaMorph (Molecular Devices)

\section{Procedure}

A. Expression and purification of ATPBP-Q105C

1. Transform Escherichia coli BL21 (DE3) cells with the plasmid encoding ATPBP-Q105C (see Note 1) as follows. Add $100 \mathrm{ng}$ plasmid to $30 \mu \mathrm{l}$ competent cells in a microtube, gently mix, and incubate on ice for $5 \mathrm{~min}$. Heat the cells at $37^{\circ} \mathrm{C}$ for $3 \mathrm{~min}$, incubate on ice for $5 \mathrm{~min}$, and add $300 \mu \mathrm{l} \mathrm{LB}$ media.

2. Plate the cells on an LB agar plate containing $100 \mu \mathrm{g} / \mathrm{ml}$ ampicillin and incubate overnight at $37^{\circ} \mathrm{C}$.

3. Inoculate $5 \mathrm{ml} 2 \times$ YT media containing $100 \mu \mathrm{g} / \mathrm{ml}$ ampicillin in a $50-\mathrm{ml}$ tube with a single colony grown on the plate, and grow the culture at $37^{\circ} \mathrm{C}$ with shaking at $200 \mathrm{rpm}$ for $6 \mathrm{~h}$.

4. Transfer $2 \mathrm{ml}$ culture to a 1-L flask with $250 \mathrm{ml} 2 \times$ YT media containing $100 \mu \mathrm{g} / \mathrm{ml}$ ampicillin, and grow the culture at $37^{\circ} \mathrm{C}$ with shaking at $200 \mathrm{rpm}$ until the $\mathrm{OD}_{600}$ reaches $0.5-0.8$.

5. Add $1 \mathrm{ml} 100 \mathrm{mM}$ IPTG to the culture and incubate at $25^{\circ} \mathrm{C}$ with shaking at $160 \mathrm{rpm}$ for $16 \mathrm{~h}$.

6. Collect the cells by centrifugation at $4^{\circ} \mathrm{C}$ for $15 \mathrm{~min}$ at $2,070 \times \mathrm{g}$, and store the cell pellet at $-80^{\circ} \mathrm{C}$.

7. Resuspend the cells in $12.5 \mathrm{ml}$ PBS on ice. Add $1.3 \mathrm{ml} \mathrm{PBS}$ containing $10 \mathrm{mg} / \mathrm{ml}$ lysozyme and perform three freeze-thaw cycles. Add $150 \mu \mathrm{l} 10 \%$ Triton X100, $150 \mu \mathrm{l} 1 \mathrm{M} \mathrm{MgCl}_{2}$, and $9 \mu \mathrm{l} 5$ $\mathrm{U} / \mu \mathrm{L}$ DNase I, and incubate at $4^{\circ} \mathrm{C}$ for $15 \mathrm{~min}$.

8. Centrifuge the cell lysate at $4^{\circ} \mathrm{C}$ for 30 min at $15,000 \times g$ and collect the supernatant in a new tube.

9. Equilibrate a HiTrap TALON crude 1-ml column with $5 \mathrm{ml} \mathrm{PBS}$, and transfer the supernatant into the column.

10. Apply $25 \mathrm{ml}$ PBS containing $10 \mathrm{mM}$ imidazole to the column for washing.

11. Apply $20 \mathrm{ml}$ PBS containing $150 \mathrm{mM}$ imidazole to the column, and collect the eluate in $1 \mathrm{ml}$ fractions in new tubes.

12. Perform SDS-PAGE analysis to identify the fractions containing ATPBP-Q105C. Measure the protein concentration by the Bradford protein assay using BSA as a standard, and calculate the molar concentration of ATPBP-Q105C (15.9 kDa) (see Note 2). 


\section{B. Fluorophore labeling}

1. Add $985 \mu \mathrm{l}$ PBS to each of two new microtubes. Transfer $10 \mu \mathrm{l}$ eluate containing $1 \mathrm{mM}$ ATPBPQ105C into each of the microtubes. Add $5 \mu 10 \mathrm{mM}$ Cy3 maleimide dissolved in DMSO to each microtube, gently mix, and incubate at $25^{\circ} \mathrm{C}$ for $45 \mathrm{~min}$.

2. Equilibrate a PD-10 desalting column with $25 \mathrm{ml}$ PBS and transfer $2 \mathrm{ml}$ mixture into the column.

3. Apply $700 \mu \mathrm{l}$ PBS to the column and discard the eluate.

4. Apply $3 \mathrm{ml}$ PBS to the column and collect the eluate containing the Cy3-labeled ATPBP-Q105C (ATPOS) in a new tube. Determine the concentration of ATPOS by the Bradford protein assay using BSA as a standard (see Note 3 ).

C. Biotinylation of ATPOS

1. Transfer $1,000 \mu \mathrm{l} 6 \mu \mathrm{M}$ ATPOS into a microtube containing $497 \mu \mathrm{l} \mathrm{PBS}$. Add $3 \mu \mathrm{l} 5 \mathrm{mM}$ NHS$\mathrm{PEG}_{4}$-biotin dissolved in DMSO, gently mix, and incubate at $25^{\circ} \mathrm{C}$ for $1 \mathrm{~h}$.

2. Equilibrate a PD-10 desalting column with $25 \mathrm{ml} P B S$ and transfer $1.5 \mathrm{ml}$ mixture into the column.

3. Apply $1.2 \mathrm{ml}$ PBS to the column and discard the eluate.

4. Apply $3 \mathrm{ml}$ PBS to the column and collect the eluate containing biotinylated ATPOS in a new tube.

5. Transfer $500 \mu \mathrm{l}$ eluate into an Amicon Ultra-0.5 centrifugal filter unit (10 kDa cutoff) and concentrate the solution by centrifugation at $4^{\circ} \mathrm{C}$ for $5 \mathrm{~min}$ at $14,000 \times \mathrm{g}$. Apply the rest of the eluate to the centrifugal filter unit and repeat the centrifugation. Determine the concentration of biotinylated ATPOS by the Bradford protein assay using BSA as a standard (see Note 4); store at $-80^{\circ} \mathrm{C}$.

D. Expression and purification of BoNT/C-Hc

1. Transform Escherichia coli BL21 (DE3) cells with the plasmid encoding BoNT/C-Hc (see Note 1) as follows. Add $100 \mathrm{ng}$ plasmid to $30 \mu \mathrm{l}$ competent cells in a microtube, gently mix, and incubate on ice for $5 \mathrm{~min}$. Heat the cells at $37^{\circ} \mathrm{C}$ for $3 \mathrm{~min}$, and incubate on ice for $5 \mathrm{~min}$. Add $100 \mu \mathrm{LB}$ media and incubate at $37^{\circ} \mathrm{C}$ for $20 \mathrm{~min}$. Plate the cells on an LB agar plate containing $20 \mu \mathrm{g} / \mathrm{ml}$ kanamycin and incubate overnight at $37^{\circ} \mathrm{C}$.

2. Inoculate $5 \mathrm{ml}$ LB media containing $20 \mu \mathrm{g} / \mathrm{ml}$ kanamycin in a $50-\mathrm{ml}$ tube with a single colony grown on the plate, and grow the culture at $37^{\circ} \mathrm{C}$ with shaking at $200 \mathrm{rpm}$ for $4 \mathrm{~h}$.

3. Transfer $2 \mathrm{ml}$ culture into a $1-\mathrm{L}$ flask with $250 \mathrm{ml} 2 \times$ YT media containing $20 \mu \mathrm{g} / \mathrm{ml}$ kanamycin, and grow the culture at $37^{\circ} \mathrm{C}$ with shaking at $200 \mathrm{rpm}$ until the $\mathrm{OD}_{600}$ reaches $0.5-1.0$.

4. Add $250 \mu \mathrm{l} 100 \mathrm{mM}$ IPTG to the culture and incubate at $25^{\circ} \mathrm{C}$ with shaking at $200 \mathrm{rpm}$ for $24 \mathrm{~h}$. Collect the cells by centrifugation at $4^{\circ} \mathrm{C}$ for $15 \mathrm{~min}$ at $2,070 \times \mathrm{g}$ and store the cell pellet at $-80^{\circ} \mathrm{C}$.

5. Resuspend the cells in $30 \mathrm{ml}$ PBS on ice and lyse the cells under a pressure of 1,200 psi with a French press. Centrifuge the cell lysate at $4^{\circ} \mathrm{C}$ for $30 \mathrm{~min}$ at $15,000 \times \mathrm{g}$ and collect the supernatant in a new tube. 
6. Equilibrate $2 \mathrm{ml}$ TALON metal affinity resin with $2 \mathrm{ml} 10 \times$ PBS in a $50-\mathrm{ml}$ tube, add the supernatant, and agitate the suspension on ice for $30 \mathrm{~min}$.

7. Transfer the suspension into an empty disposable PD-10 column and apply $50 \mathrm{ml}$ PBS containing $5 \mathrm{mM}$ imidazole to the column for washing.

8. Apply $5 \mathrm{ml}$ PBS containing $150 \mathrm{mM}$ imidazole to the column and collect the eluate in $1 \mathrm{ml}$ fractions in new tubes. Measure the protein concentration by the Bradford protein assay using BSA as a standard; determine the fraction containing the highest concentration of the protein (designated $1^{\text {st }}$ fraction), the fraction containing the second highest concentration of the protein (designated $2^{\text {nd }}$ fraction), and the fraction containing the third highest concentration of the protein (designated $3^{\text {rd }}$ fraction) (see Note 5).

9. Equilibrate a PD-10 desalting column with $25 \mathrm{ml}$ PBS and transfer $2 \mathrm{ml} 1^{\text {st }}$ and $2^{\text {nd }}$ fractions and $500 \mu \mathrm{l} 3^{\text {rd }}$ fraction into the column.

10. Apply $500 \mu \mathrm{l} 3^{\text {rd }}$ fraction and $2.6 \mathrm{ml}$ PBS to the column and collect $3.1 \mathrm{ml}$ eluate containing BoNT/C-Hc in a new tube. Determine the concentration of BoNT/C-Hc by the Bradford protein assay using BSA as a standard and calculate the molar concentration of BoNT/C-Hc (51.6 kDa) (see Note 6).

E. Biotinylation of BoNT/C-Hc

1. Add $750 \mu \mathrm{l}$ PBS to each of two new microtubes. Transfer $250 \mu \mathrm{l}$ eluate containing $40 \mu \mathrm{M}$ BoNT/C-Hc into each of the microtubes. Add $1 \mu \mathrm{l} 200$ mM NHS-PEG 4 -biotin dissolved in DMSO to each microtube, gently mix, and incubate on ice for $45 \mathrm{~min}$.

2. Equilibrate a PD-10 desalting column with $25 \mathrm{ml}$ PBS and transfer $2 \mathrm{ml}$ mixture into the column.

3. Apply $500 \mu \mathrm{l}$ PBS to the column and discard the eluate.

4. Apply $3.1 \mathrm{ml}$ PBS to the column and collect the eluate containing biotinylated BoNT/C-Hc in a new tube.

5. Transfer $500 \mu \mathrm{l}$ eluate into an Amicon Ultra- 0.5 centrifugal filter unit (30 kDa cutoff) and concentrate the solution by centrifugation at $4^{\circ} \mathrm{C}$ for $5 \mathrm{~min}$ at $14,000 \times \mathrm{g}$. Apply the rest of the eluate to the centrifugal filter unit in $500 \mu \mathrm{l}$ aliquots and repeat the centrifugation. Determine the concentration of biotinylated BoNT/C-Hc by the Bradford protein assay using BSA as a standard (see Note 7); store at $-80^{\circ} \mathrm{C}$.

F. Surgery

1. Anesthetize a male C57BL/6 mouse by intraperitoneally injecting $0.1 \mathrm{ml} / 10 \mathrm{~g}$ body weight of anesthetic solution. Wait for 5-10 min and then assess the depth of anesthesia by tail pinch. Place the mouse under a wide-field microscope and maintain the rectal temperature at $37^{\circ} \mathrm{C}$ with a heating pad.

2. Remove the scalp covering the skull with scissors, attach a custom-made metal frame to the exposed skull with dental acrylic (Figure 2A), and fix the metal frame with a custom-made frame holder (Figure 2B). 
3. Make a groove in the skull with a dental drill along the circumference of a 4-mm-diameter circle centered $3 \mathrm{~mm}$ posterior to the bregma and $3 \mathrm{~mm}$ lateral to the midline. Remove the bone flap within the circle with forceps, and leave the dura intact. Cover the surface of the exposed cortex with aCSF.

A

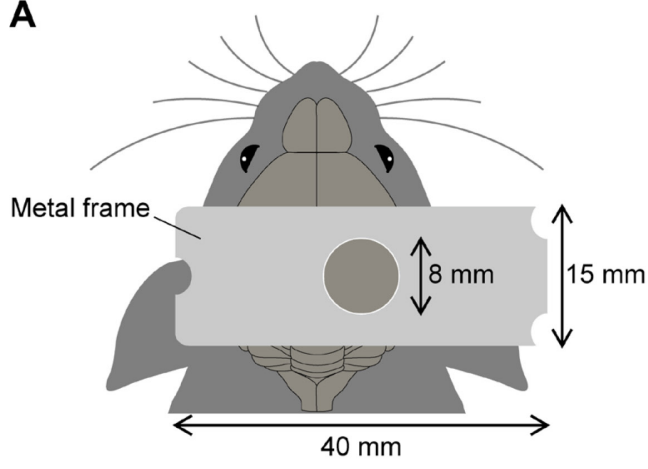

C

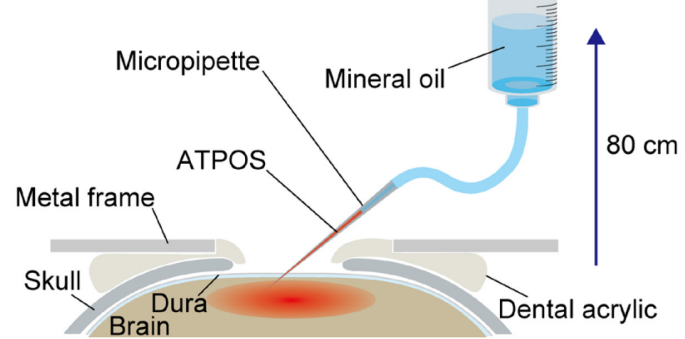

B

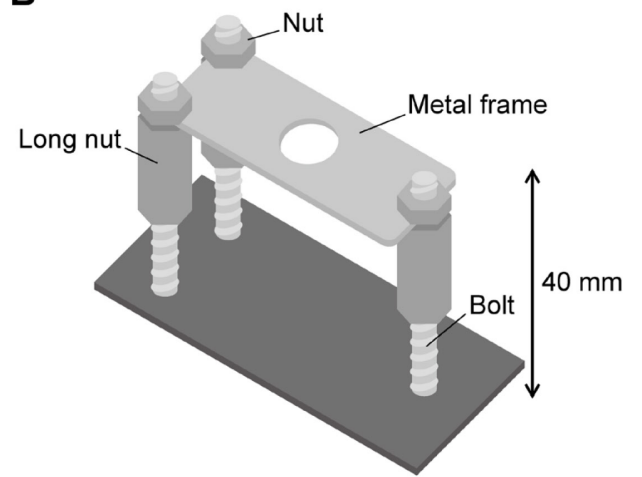

Figure 2. Schematic illustration of the experimental set-up for the application of ATPOS to the mouse cerebral cortex. A. A custom-made metal frame is attached to the mouse skull. B. A custom-made frame holder. The metal frame is clamped at three positions by the upper nuts and the lower long nuts. C. ATPOS is pressure-injected into the cerebral cortex with a micropipet through the craniotomy by raising the cylinder containing mineral oil by $80 \mathrm{~cm}$ vertically from the pipet tip.

\section{G. Injection of ATPOS}

1. Prepare a glass micropipet as follows. Pull a glass capillary on a P-97 pipet puller and bevel the tip of the micropipet at an angle of 35 degrees relative to a grinding plate until the inner tip diameter reaches $10 \mu \mathrm{m}$.

2. Prepare the ATPOS complex solution as follows. Transfer $3 \mu \mathrm{l} 40 \mu \mathrm{M}$ biotinylated ATPOS into a microtube containing $15.5 \mu \mathrm{lBS}$, and add $1 \mu \mathrm{l} 40 \mu \mathrm{M}$ biotinylated BoNT/C-Hc. Add $0.5 \mu \mathrm{l} 83$ $\mu \mathrm{M}$ Alexa488-labeled streptavidin dissolved in PBS and gently mix.

3. Load $20 \mu$ ITPOS complex solution into the micropipet from its back end with a microloader pipet tip, and fill the rest of the inner space of the micropipet with $10 \mu \mathrm{l}$ mineral oil. Attach the external cylinder of a $10-\mathrm{ml}$ syringe to a $1.5-\mathrm{m}$-long plastic tube and add $5 \mathrm{ml}$ mineral oil to the cylinder to fill the plastic tube. Connect the other end of the plastic tube to the back end of the micropipet (see Note 8). 
4. Fix the micropipette in a stereotaxic manipulator at an angle of 45 degrees relative to the brain surface and insert the pipette tip to a depth of $300 \mu \mathrm{m}$ from the brain surface.

5. Inject the ATPOS complex solution at $1 \mathrm{psi}$ by raising the cylinder $80 \mathrm{~cm}$ vertically from the pipet tip for 20-30 min (Figure 2C).

6. After the injection is finished, withdraw the micropipet from the brain.

\section{$\mathrm{H}$. In vivo ATP imaging}

1. Find an appropriate imaging area by observing the fluorescence of ATPOS under 555-nm illumination, and focus on the brain surface by observing a brightfield image of the imaging area.

2. Acquire Cy 3 and Alexa488 fluorescence images with an EM-CCD camera at $2.5 \mathrm{~Hz}$ sequentially (200 ms duration for each fluorescence) by alternating 555-nm and 490-nm illumination for excitation of Cy3 and Alexa488, respectively (see Note 9).

3. For drug application, load an appropriate volume of the drug dissolved in aCSF into a glass micropipet, and fill the rest of the inner space of the micropipet with mineral oil (see Note 10). Connect the micropipet with a plastic tube filled with mineral oil and inject the drug at 1 psi by raising the cylinder attached to the plastic tube $80 \mathrm{~cm}$ vertically from the pipet tip.

4. To evoke CSD by electrical stimulation, fix a monopolar tungsten microelectrode in a stereotaxic manipulator at an angle of 45 degrees relative to the brain surface and place the tip of the electrode below the dura. Attach a ground wire to the custom-made metal frame for head fixation, and deliver a train of $100 \mu$ s pulses at $200 \mathrm{~Hz}$ lasting for $1 \mathrm{~s}$ with an intensity of $10 \mathrm{~mA}$ with a stimulus isolator.

\section{Data analysis}

1. Open a series of acquired images using the ImageJ/Fiji software (ver. 1.50e), and split the images into a stack from the Cy3 fluorescence channel and a stack from the Alexa488 fluorescence channel (Figure 3A).

2. Calculate the background intensity for each fluorescence channel as the mean pixel values in the cortical area that is not stained with the ATPOS complex, and subtract the background intensity from the stack of each fluorescence channel (Figure 3B).

3. Process the stacks using a Gaussian spatial filter with a radius of 2 pixels (pixel size $=8 \mu \mathrm{m}$ ) (Figure $3 \mathrm{C}$ ), and create a stack of ratiometric images by dividing the stack of the Cy3 fluorescence channel by that of the Alexa488 fluorescence channel (Figure 3D).

4. Calculate the ratiometric fluorescence response of ATPOS $\left(R / R_{0}\right)$ as follows. Create an image showing the baseline of the fluorescence ratio $\left(R_{0}\right)$ by averaging the ratiometric images acquired before drug application or electrical stimulation (Figure 3E). Divide the stack of ratiometric images by the averaged image (Figure $3 F$ ) and plot a time-course of the mean fluorescence response in regions of interest (ROIs) selected in the cortical area stained with the ATPOS complex (Figure 4). 
A

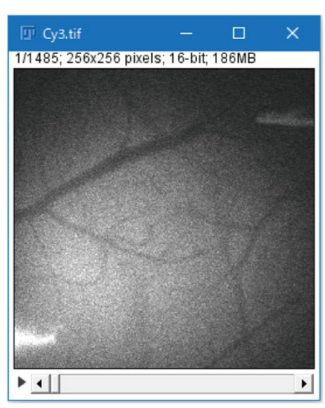

C

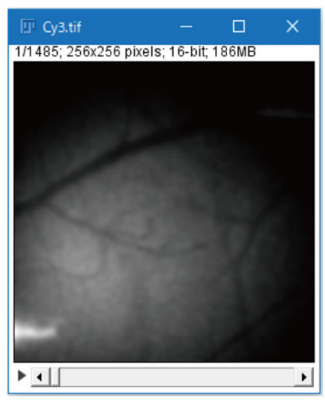

$\mathbf{E}$

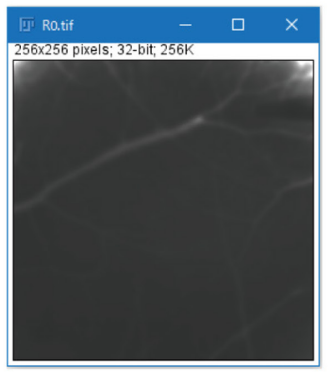

B
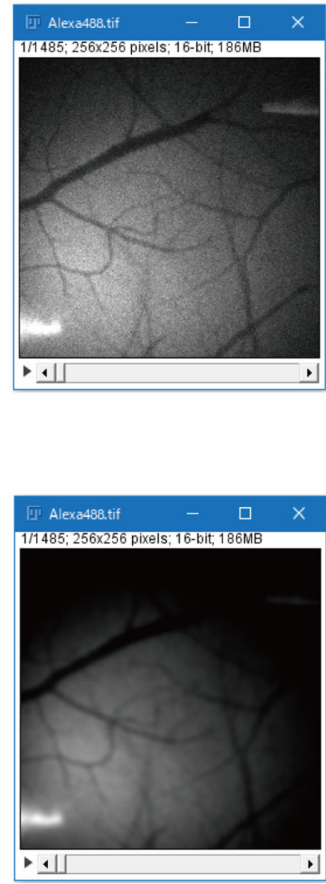

.11

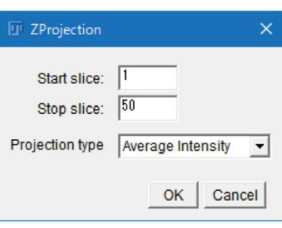

D

$\mathbf{F}$
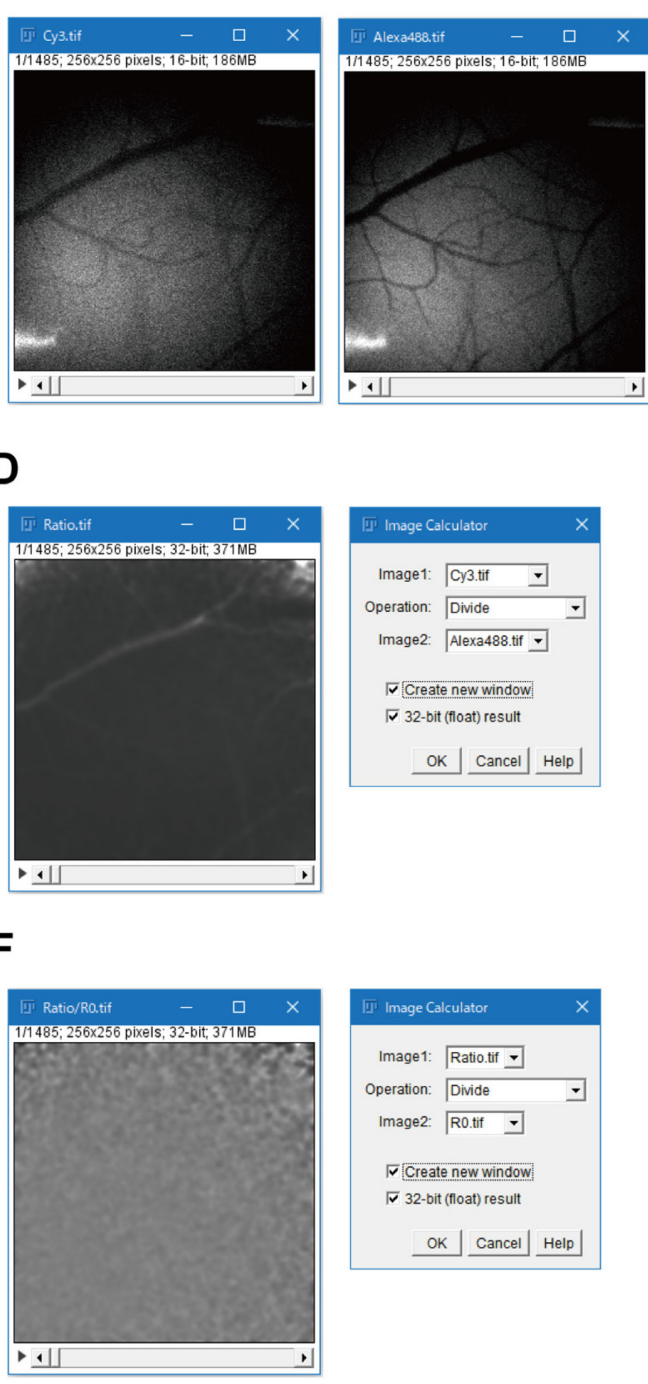

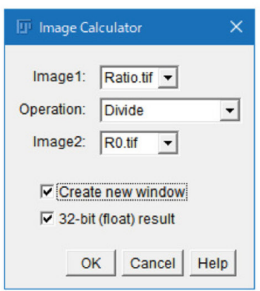

OK」 Cancel $\underline{\text { Help }}$

Figure 3. Image processing with the ImageJ/Fiji software. A. Stacks of Cy3 (left) and Alexa488 (right) fluorescence channels obtained by splitting channels using menu commands (Image > Stacks > Tools > Deinterleave). B. Stacks of Cy3 (left) and Alexa488 (right) fluorescence channels obtained by subtraction of the background fluorescence using menu commands (Process > Math > Subtract). C. Stacks of Cy3 (left) and Alexa488 (right) fluorescence channels obtained by Gaussian-spatial filtering using menu commands (Process $>$ Filters > Gaussian Blur). D. A stack of ratiometric images (left) obtained by division of the stack of the Cy3 fluorescence channel by that of the Alexa488 fluorescence channel (right) using menu commands (Process $>$ Image Calculator). E. A ratiometric image $\left(R_{0}\right)$ (left) obtained by averaging frames acquired during the baseline period (right) using menu commands (Image > Stacks $>$ Z Project). Enter frame numbers at the start and end of the baseline period in the 'Start slice' and 'Stop slice' boxes, respectively. In this example, the baseline period is $20 \mathrm{~s}$ (400 ms per frame $\times 50$ frames). F. A stack of images $\left(R / R_{0}\right)$ (left) obtained by dividing the stack of ratiometric images by the averaged image (right) using menu commands (Process $>$ Image Calculator). 
A

B

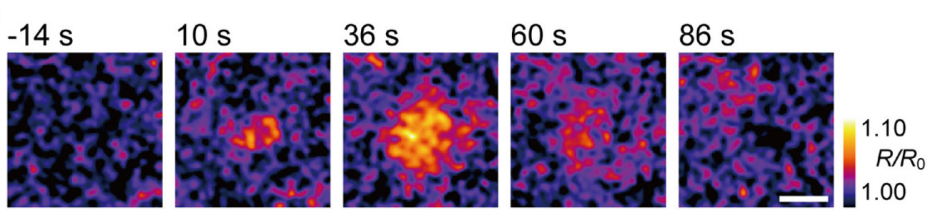

C

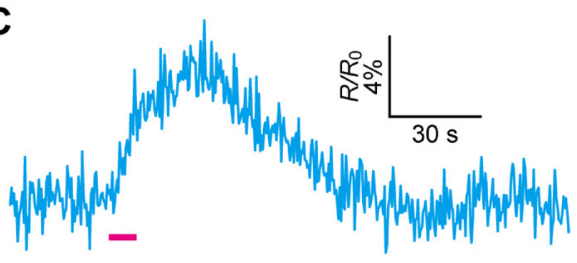

D $\quad E$

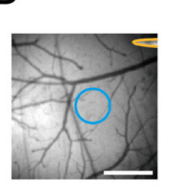

E -20 s
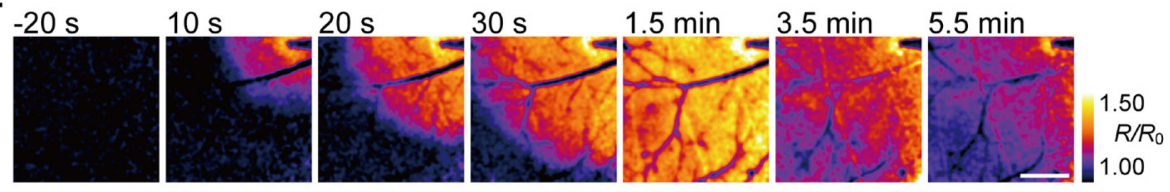

$\mathbf{F}$

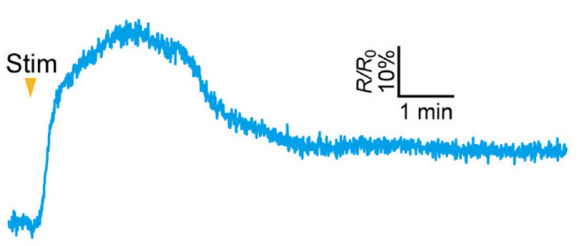

Figure 4. In vivo fluorescence imaging of extracellular ATP in the cerebral cortex. A-C. Visualization of the elevation of extracellular ATP levels after application of $10 \mathrm{mM}$ ATP. A. The position of the micropipet for ATP injection (magenta) and the location of an ROI with a 15-pixel radius (blue). B. Representative images showing the ratiometric fluorescence response of ATPOS $\left(R / R_{0}\right)$ upon ATP injection. The extracellular ATP levels are increased around the micropipet. Time after the start of injection is presented above the images. Scale bar, $250 \mu \mathrm{m}$. C. A time-course of the ratiometric fluorescence response of ATPOS $\left(R / R_{0}\right)$ extracted from the ROI depicted in A. The magenta bar indicates ATP injection. D-F. Visualization of wave-like extracellular ATP dynamics during CSD evoked by electrical stimulation. D. The position of the stimulating electrode (yellow) and the location of an $\mathrm{ROI}$ with a 25-pixel radius (blue). E. Representative images showing the ratiometric fluorescence response of ATPOS $\left(R / R_{0}\right)$ upon electrical stimulation. A wave-like extracellular ATP release propagates from the stimulation site. Time after the initiation of electrical stimulation is presented above the images. Scale bar, 500 $\mu \mathrm{m}$. F. Time-course of the ratiometric fluorescence response of ATPOS $\left(R / R_{0}\right)$ extracted from the ROI depicted in $\mathrm{D}$.

\section{$\underline{\text { Notes }}$}

1. The bacterial expression construct is based on $\mathrm{pET}$ vectors in which the recombinant protein is expressed under the control of a T7 promotor. Therefore, one has to use a host strain bearing DE3 lysogen, such as BL21(DE3), with which IPTG induces the expression of T7 polymerase, 
leading to activation of the $\mathrm{T7}$ promotor.

2. The molar concentration of the purified ATPBP-Q105C is typically around $1 \mathrm{mM}$.

3. The molar concentration of ATPOS is typically around $6 \mu \mathrm{M}$.

4. The molar concentration of biotinylated ATPOS is typically around $40 \mu \mathrm{M}$.

5. The protein concentration in the $1^{\text {st }}, 2^{\text {nd }}$, and $3^{\text {rd }}$ fraction is typically around $3 \mathrm{mg} / \mathrm{ml}, 2 \mathrm{mg} / \mathrm{ml}$ and $1 \mathrm{mg} / \mathrm{ml}$, respectively.

6. The molar concentration of BoNT/C-Hc is typically around $40 \mu \mathrm{M}$.

7. The molar concentration of biotinylated BoNT/C-Hc is typically around $40 \mu \mathrm{M}$.

8. It is important to prevent air bubbles getting into the micropipet or plastic tube.

9. The obtained images have a dimension of $256 \times 256$ pixels, with a pixel size of $8 \mu \mathrm{m} \times 8 \mu \mathrm{m}$ at the object plane.

10. For application of ATP, load $20 \mu \mathrm{l} 10 \mathrm{mM}$ ATP dissolved in aCSF into a glass micropipet and fill the rest of the inner space of the micropipet with $10 \mu \mathrm{l}$ mineral oil.

\section{$\underline{\text { Recipes }}$}

1. LB agar plates

$10 \mathrm{~g}$ tryptone

$5 \mathrm{~g}$ yeast extract

$5 \mathrm{~g} \mathrm{NaCl}$

$1 \mathrm{~g}$ glucose

$15 \mathrm{~g}$ agar

Add Milli-Q $\mathrm{H}_{2} \mathrm{O}$ to $1 \mathrm{~L}$

2. $2 \times$ YT media

$16 \mathrm{~g}$ tryptone

$10 \mathrm{~g}$ yeast extract

$5 \mathrm{~g} \mathrm{NaCl}$

Add Milli-Q $\mathrm{H}_{2} \mathrm{O}$ to $1 \mathrm{~L}$

3. LB media

$10 \mathrm{~g}$ tryptone

$5 \mathrm{~g}$ yeast extract

$5 \mathrm{~g} \mathrm{NaCl}$

$1 \mathrm{~g}$ glucose

Add Milli-Q $\mathrm{H}_{2} \mathrm{O}$ to $1 \mathrm{~L}$

4. $\mathrm{PBS}(\mathrm{pH} 7.4)$

$8 \mathrm{~g} \mathrm{NaCl}$

$0.2 \mathrm{~g} \mathrm{KCl}$

$0.2 \mathrm{~g} \mathrm{KH}_{2} \mathrm{PO}_{4}$

$2.9 \mathrm{~g} \mathrm{Na}_{2} \mathrm{HPO}_{4} \cdot 12 \mathrm{H}_{2} \mathrm{O}$ 
Add Milli-Q $\mathrm{H}_{2} \mathrm{O}$ to $1 \mathrm{~L}$

5. $10 \times \mathrm{PBS}(\mathrm{pH} 7.4)$

$80 \mathrm{~g} \mathrm{NaCl}$

$2 \mathrm{~g} \mathrm{KCl}$

$2 \mathrm{~g} \mathrm{KH}_{2} \mathrm{PO}_{4}$

$29 \mathrm{~g} \mathrm{Na}_{2} \mathrm{HPO}_{4} \cdot 12 \mathrm{H}_{2} \mathrm{O}$

Add Milli-Q $\mathrm{H}_{2} \mathrm{O}$ to $1 \mathrm{~L}$

6. aCSF

$7.3 \mathrm{~g} \mathrm{NaCl}$

$0.3 \mathrm{~g} \mathrm{KCl}$

$0.2 \mathrm{~g} \mathrm{NaH}_{2} \mathrm{PO}_{4} \cdot 2 \mathrm{H}_{2} \mathrm{O}$

$2.2 \mathrm{~g} \mathrm{NaHCO}_{3}$

$0.3 \mathrm{~g} \mathrm{CaCl}_{2} \cdot 2 \mathrm{H}_{2} \mathrm{O}$

$0.2 \mathrm{~g} \mathrm{MgCl}_{2} \cdot 6 \mathrm{H}_{2} \mathrm{O}$

$3.6 \mathrm{~g}$ glucose

Add Milli-Q $\mathrm{H}_{2} \mathrm{O}$ to $1 \mathrm{~L}$

7. HBS

$2.4 \mathrm{~g}$ HEPES, $8.8 \mathrm{~g} \mathrm{NaCl}, 0.2 \mathrm{~g} \mathrm{KCl}$. Add Milli-Q $\mathrm{H}_{2} \mathrm{O}$ to $1 \mathrm{~L}$

8. Anesthetic solution

$1.9 \mathrm{ml}$ medetomidine $(1 \mathrm{mg} / \mathrm{ml})$

$2.0 \mathrm{ml}$ midazolam $(5 \mathrm{mg} / \mathrm{ml})$

$2.5 \mathrm{ml}$ butorphanol $(5 \mathrm{mg} / \mathrm{ml})$

Add $0.9 \% \mathrm{NaCl}$ to $25 \mathrm{ml}$

\section{Acknowledgments}

This work was supported by Grants-in-Aid for Scientific Research (KAKENHI) from the Ministry of Education, Culture, Sports, Science, and Technology of Japan (MEXT) (18K14915 to H. Sekiya, 17H04764 and 18H04726 to D.A., 19K16251 to H. Sakamoto, 17K08584 to S.N., 25221304 to M.I., and $17 \mathrm{H} 04029$ and 19K22247 to K. H.), Japan Science and Technology Agency (PRESTO, JPMJPR17P1 to D.A.), and Takeda Science Foundation (to N.K.). This protocol was adapted from previous work (Kitajima et al., 2020).

\section{Competing interests}

The authors declare no conflicts of interest. 
Please cite this article as: Kitajima, N. et al., (2021). In vivo Fluorescence Imaging of Extracellular ATP in the Mouse Cerebral Cortex with a Hybrid-type Optical Sensor. Bio-protocol 11(11): e4046. DOI: 10.21769/BioProtoc.4046.

\section{Ethics}

All experimental procedures used in animal experiments have been approved by the Animal Welfare Committee of the University of Tokyo.

\section{References}

1. Arai, S., Kriszt, R., Harada, K., Looi, L. S., Matsuda, S., Wongso, D., Suo, S., Ishiura, S., Tseng, Y. H., Raghunath, M., Ito, T., Tsuboi, T. and Kitaguchi, T. (2018). RGB-Color Intensiometric Indicators to Visualize Spatiotemporal Dynamics of ATP in Single Cells. Angew Chem Int Ed Engl 57(34): 10873-10878.

2. Berg, J., Hung, Y. P. and Yellen, G. (2009). A genetically encoded fluorescent reporter of ATP:ADP ratio. Nat Methods 6 (2): 161-166.

3. Burnstock, G. (2007). Physiology and pathophysiology of purinergic neurotransmission. Physiol Rev 87 (2): 659-797.

4. Butt, A. M. (2011). ATP: A ubiquitous gliotransmitter integrating neuron-glial networks. Semin Cell Dev Biol 22 (2): 205-213.

5. Chesler, M., and Kaila, K. (1992). Modulation of pH by neuronal activity. Trends Neurosci 15 (10): 396-402.

6. Chudakov, D. M., Matz, M. V., Lukyanov, S. and Lukyanov, K. A. (2010). Fluorescent proteins and their applications in imaging living cells and tissues. Physiol Rev 90 (3): 1103-1163.

7. Conley, J. M., Radhakrishnan, S., Valentino, S. A. and Tantama, M. (2017). Imaging extracellular ATP with a genetically-encoded, ratiometric fluorescent sensor. PLoS One 12 (11): e0187481.

8. Fields, R. D. and Burnstock, G. (2006). Purinergic signalling in neuron-glia interactions. Nat Rev Neurosci 7 (6): 423-436.

9. Giepmans, B. N. G., Adams, S. R., Ellisman, M. H. and Tsien, R. Y. (2006). The fluorescent toolbox for assessing protein location and function. Science 312 (5771): 217-224.

10. Gourine, A. V., Llaudet, E., Dale, N. and Spyer, K. M. (2005). ATP is a mediator of chemosensory transduction in the central nervous system. Nature 436 (7047): 108-111.

11. Heinrich, A., Andó, R. D., Túri, G., Rózsa, B. and Sperlágh, B. (2012). $\underline{K^{+} \text {depolarization evokes }}$ ATP, adenosine and glutamate release from glia in rat hippocampus: a microelectrode biosensor study. Br J Pharmacol 167 (5): 1003-1020.

12. Imamura, H., Huynh Nhat, K. P., Togawa, H., Saito, K., lino, R., Kato-Yamada, Y., Nagai, T. and Noji, H. (2009). Visualization of ATP levels inside single living cells with fluorescence resonance energy transfer-based genetically encoded indicators. Proc Natl Acad Sci 106 (37): $15651-15656$.

13. Khakh, B. S. and North, R. A. (2012). Neuromodulation by extracellular ATP and P2X receptors in the CNS. Neuron 76 (1): 51-69. 
Please cite this article as: Kitajima, N. et al., (2021). In vivo Fluorescence Imaging of Extracellular ATP in the Mouse Cerebral Cortex with a Hybrid-type Optical Sensor. Bio-protocol 11(11): e4046. DOI: 10.21769/BioProtoc.4046.

14. Kitajima, N., Takikawa, K., Sekiya, H., Satoh, K., Asanuma, D., Sakamoto, H., Takahashi, S., Hanaoka, K., Urano, Y., Namiki, S., lino, M. and Hirose, K. (2020). Real-time in vivo imaging of extracellular ATP in the brain with a hybrid-type fluorescent sensor. Elife 9: : e57544.

15. Lauritzen, M., Dreier, J. P., Fabricius, M., Hartings, J. A., Graf, R. and Strong, A. J. (2011). Clinical relevance of cortical spreading depression in neurological disorders: migraine, malignant stroke, subarachnoid and intracranial hemorrhage, and traumatic brain injury. $J$ Cereb Blood Flow Metab 31 (1): 17-35.

16. Lobas, M. A., Tao, R., Nagai, J., Kronschläger, M. T., Borden, P. M.., Marvin, J. S., Looger, L. L. and Khakh, B. S. (2019). A genetically encoded single-wavelength sensor for imaging cytosolic and cell surface ATP. Nat Commun 10 (1): 711.

17. MacVicar, B. A. and Newman, E. A. (2015). Astrocyte regulation of blood flow in the brain. Cold Spring Harb Perspect Biol 7 (5): a020388.

18. Magnotta, V. A., Heo, H. Y., Dlouhy, B. J., Dahdaleh, N. S., Follmer, R. L., Thedens, D. R., Welsh, M. J. and Wemmie, J. A. (2012). Detecting activity-evoked pH changes in human brain. Proc Natl Acad Sci U S A 109 (21): 8270-8273.

19. Melani, A., Turchi, D., Vannucchi, M. G., Cipriani, S., Gianfriddo, M. and Pedata, F. (2005). ATP extracellular concentrations are increased in the rat striatum during in vivo ischemia. Neurochem Int 47 (6): 442-448.

20. Pankratov, Y., Lalo, U., Verkhratsky, A. and North, R. A. (2006). Vesicular release of ATP at central synapses. Pflugers Arch 452 (5): 589-597.

21. Schock, S. C., Munyao, N., Yakubchyk, Y., Sabourin, L. A., Hakim, A. M., Ventureyra, E. C. G. and Thompson, C. S. (2007). Cortical spreading depression releases ATP into the extracellular space and purinergic receptor activation contributes to the induction of ischemic tolerance. Brain Res 1168: 129-138.

22. Tantama, M., Martínez-François, J. R., Mongeon, R. and Yellen, G. (2013). Imaging energy status in live cells with a fluorescent biosensor of the intracellular ATP-to-ADP ratio. Nat Commun 4 (1): 2550.

23. Takikawa, K., Asanuma, D., Namiki, S., Sakamoto,H., Ariyoshi, T., Kimpara, N. and Hirose, K. (2014). High - Throughput Development of a Hybrid - Type Fluorescent Glutamate Sensor for Analysis of Synaptic Transmission. Angew Chem Int Ed Engl 53 (49): 13439-13443.

24. Tsien, R. Y. (1998). The green fluorescent protein. Annu Rev Biochem 67 (1): 509-544.

25. Tsukamoto, K., Kohda, T., Mukamoto, M., Takeuchi, K., Ihara, H., Saito, M. and Kozaki, S. (2005). Binding of Clostridium botulinum type $C$ and $D$ neurotoxins to ganglioside and phospholipid. J Biol Chem 280 (42): 35164-35171.

26. Yaginuma, H., Kawai, S., Tabata, K. V., Tomiyama, K., Kakizuka, A., Komatsuzaki, T., Noji, H., and Imamura, H. (2015). Diversity in ATP concentrations in a single bacterial cell population revealed by quantitative single-cell imaging. Sci Rep 4 (1): 6522. 
Please cite this article as: Kitajima, N. et al., (2021). In vivo Fluorescence Imaging of Extracellular ATP in the Mouse Cerebral Cortex with a Hybrid-type Optical Sensor. Bio-protocol 11(11): e4046. DOI: 10.21769/BioProtoc.4046.

27. Yegutkin, G. G. (2008). Nucleotide- and nucleoside-converting ectoenzymes: Important modulators of purinergic signalling cascade. Biochim Biophys Acta - Mol Cell Res 1783 (5): 673694.

28. Zimmermann, H. (2016). Extracellular ATP and other nucleotides-ubiquitous triggers of intercellular messenger release. Purinergic Signal 12 (1): 25-57. 